\title{
Evaluation of rolling shear modulus and strength of Japanese cedar cross-laminated timber (CLT) laminae
}

\author{
Seiichiro Ukyo* (10, Kenta Shindo and Atsushi Miyatake
}

\begin{abstract}
Cross-laminated timber (CLT) panels are fabricated with their layers stacked crosswise. Owing to the low shear modulus and strength in the rolling shear direction, the shear properties of cross-layers influence the overall deflection and shear capacities of CLT panels. The aim of the present study is to determine the rolling shear properties of Japanese cedar and investigate how annual ring patterns and lamina geometry influence shear properties. Using a test configuration similar to the standard shear test configuration prescribed in European Standards (EN 408), a single lamina shear test was conducted. To investigate the influence of thickness-to-width aspect ratio of the lamina on the rolling shear properties, samples with three different widths, including 62,88 , and $112 \mathrm{~mm}$, with a constant thickness of $24 \mathrm{~mm}$ were tested. The geometrical features of the annual ring patterns of each test sample were measured. The mean rolling shear moduli were 72,91 , and $109 \mathrm{MPa}$, and the mean rolling shear strengths were 1.54, 1.83, and $2.02 \mathrm{MPa}$ for the 62-, 88-, and 112-mm sample widths, respectively. Shear strength was highly correlated with shear modulus. The mean shear modulus and strength, in addition to the $5 \%$ quantile, increased with an increase in lamina width. Across all sample widths, rolling shear modulus and strength decreased with an increasing radial distance from the pith. Using the principle of continuum mechanics, the influence of the annual ring angle relative to the shear force direction was examined quantitatively using the finite element method. The results suggest that shear modulus and its variance are influenced greatly by the annual ring structure.
\end{abstract}

Keywords: Rolling shear, Cross-laminated timber, Annual ring

\section{Introduction}

Cross-laminated timber (CLT) panels are fabricated with layers stacked crosswise. When the panels are subjected to close out-of-plane loads, a critical shear stress is introduced to the cross-layers. Owing to the low shear modulus and strength in the rolling shear direction of the laminae, the cross-layers influence the overall deflection and shear capacities of the CLT panels. Extensive studies have been conducted on the rolling shear properties in relation to laminae sizes. Aicher and Dill-langer [1] studied the effects of sawing patterns on rolling shear modulus using polar anisotropic finite element analysis. They concluded that the shear modulus at the macro-level

*Correspondence: ukyo@ffpriaffrc.go.jp

Forestry and Forest Products Research Institute, Matsunosato 1, Tsukuba, Ibaraki, Japan (i.e., lamina size) is not an intrinsic material property, but a global apparent quantity that is highly dependent on the sawing pattern. Ehrhart et al. [2] studied the rolling shear properties of single lamina prepared from various European timber species using a shear test configuration similar to the EN 408 timber test standard [3]. They conducted a parametric study on Norway spruce to assess the effect of various radial distances from the pith (i.e., sawing pattern) and aspect ratios of laminae on the rolling shear properties. In addition, Aicher et al. [4] investigated the influence of different sawing patterns on the rolling shear properties of beech. The annual ring patterns were classified into four established sawing patterns based on the mathematical descriptions of the orientation of the annual rings.

Japanese cedar is one of the most common species used in the production of CLT in Japan. Okabe et al. [5] 
determined the rolling shear strength of Japanese cedar single lamina using a compression test on a three-layered CLT that had a geometry similar to the test configuration specified in European Standards (EN 314-1) [6].

The goal of the present study is to identify the rolling shear properties of Japanese cedar and investigate the influence of the annual ring pattern and lamina geometry on the shear properties. A single lamina shear test was conducted using the test configuration specified in the EN 408 test standard to test the rolling shear properties.

\section{Materials and methods}

Laminae prepared from Japanese cedar (Cryptomeria japonica) boards with an elastic modulus $E_{\mathrm{fr}}$ within the 6.3-9.8 GPa range in a direction parallel to the grain were extracted as test specimen. The elastic modulus was measured using a longitudinal vibration test. The crosssection of the original lamina was $112 \mathrm{~mm}$ wide and $25 \mathrm{~mm}$ thick.

To investigate the effects of thickness-to-width aspect ratio of laminae on the rolling shear properties, samples with three widths, including 62, 88, and $112 \mathrm{~mm}$, with a constant length of $100 \mathrm{~mm}$ in the longitudinal direction were cut from the same lamina. To minimize heterogeneity in the material along the longitudinal direction, the samples were obtained from a section that did not contain knots or other types of defects. Therefore, the three samples were not closely matched in the original lamina. In total, 28 samples were prepared for each width group. Because the main objective was to assess the effect of cross-sectional aspect ratio, laminae with relatively symmetrical annual ring patterns were selected for the sets of samples. To include samples where the pith was located eccentrically along the width direction, 15 additional samples in which the annual ring patterns in the crosssection were asymmetrical were prepared (for the 112mm width).

Images of the cross-section of each sample were captured using a flatbed scanner to measure the geometrical features of the annual ring patterns. Circles were fitted onto the points on the annual ring using the "fitcircle.m" MATLAB function [7]. The function fits a circle to a set of points while minimizing geometrical error using nonlinear least squares [8]. The median value of the central coordinates obtained from multiple annual rings was determined to be the location of the pith. Figure 1 illustrates the definition of the distances, the radial distance to the pith $(V)$, and the tangential distance to the pith $(H)$.

As illustrated in Fig. 2, 38-mm-thick hardwood plates were glued onto both sides of the samples using a polyvinyl acetate emulsion. To provide a smooth surface for the adhesive, both faces of the samples were planed to a thickness of $24 \mathrm{~mm}$. The ends of the hardwood plates

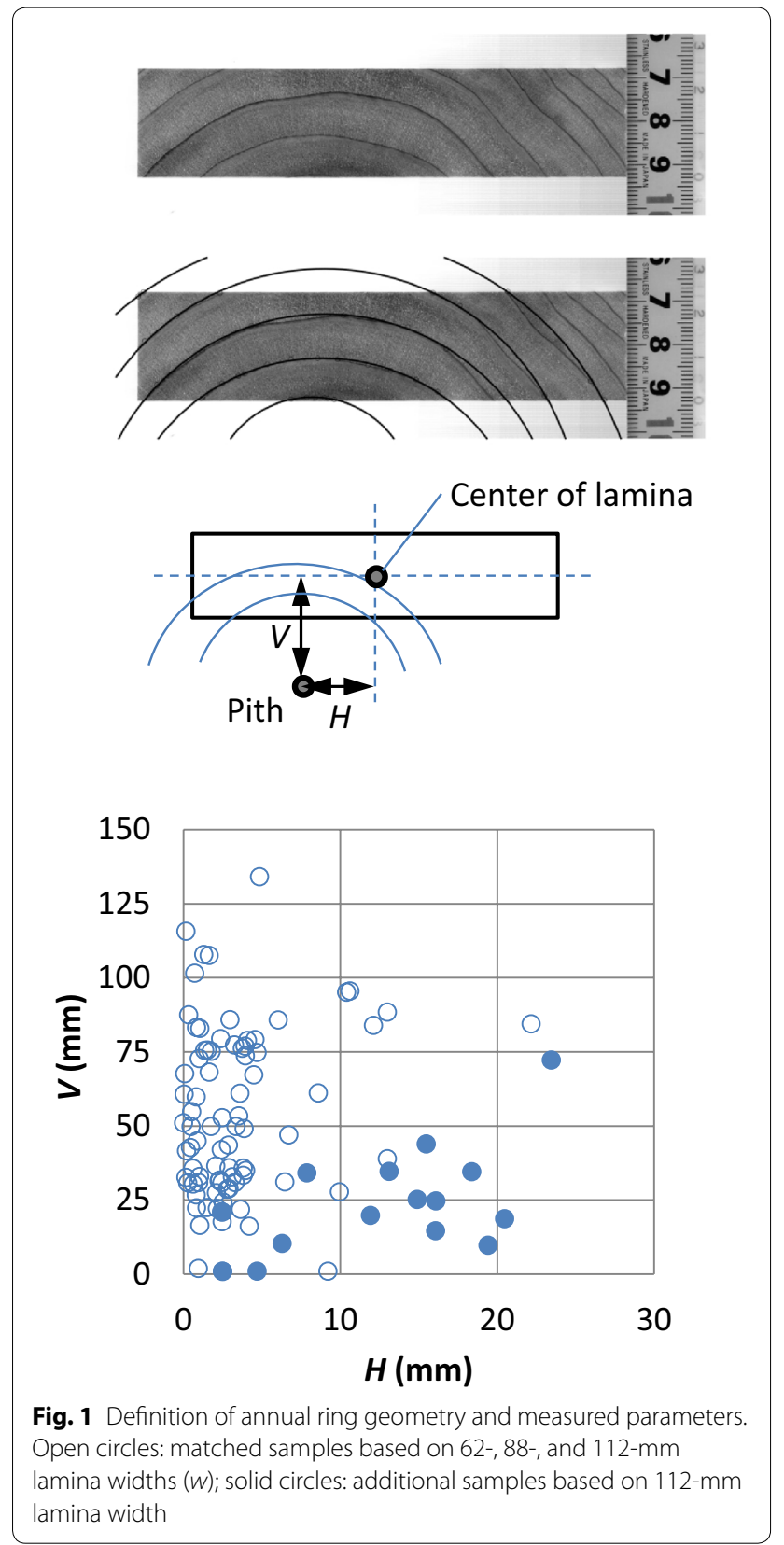

were cut so that the loading direction and the direction parallel to the shear plane formed an angle of $14^{\circ}$. Prior to testing, all samples were conditioned at room temperature $\left(20^{\circ} \mathrm{C}\right)$ with $60 \%$ relative humidity. Moisture content (MC) was measured using matched samples using the oven-dry method. MC was in the $8.9-11.8 \%$ range, with a mean of $10 \%$.

To measure displacement and strain distribution using digital image correlation methods, speckle patterns were added on the surfaces of the samples. To achieve a high contrast speckle pattern, the surface of each sample was 

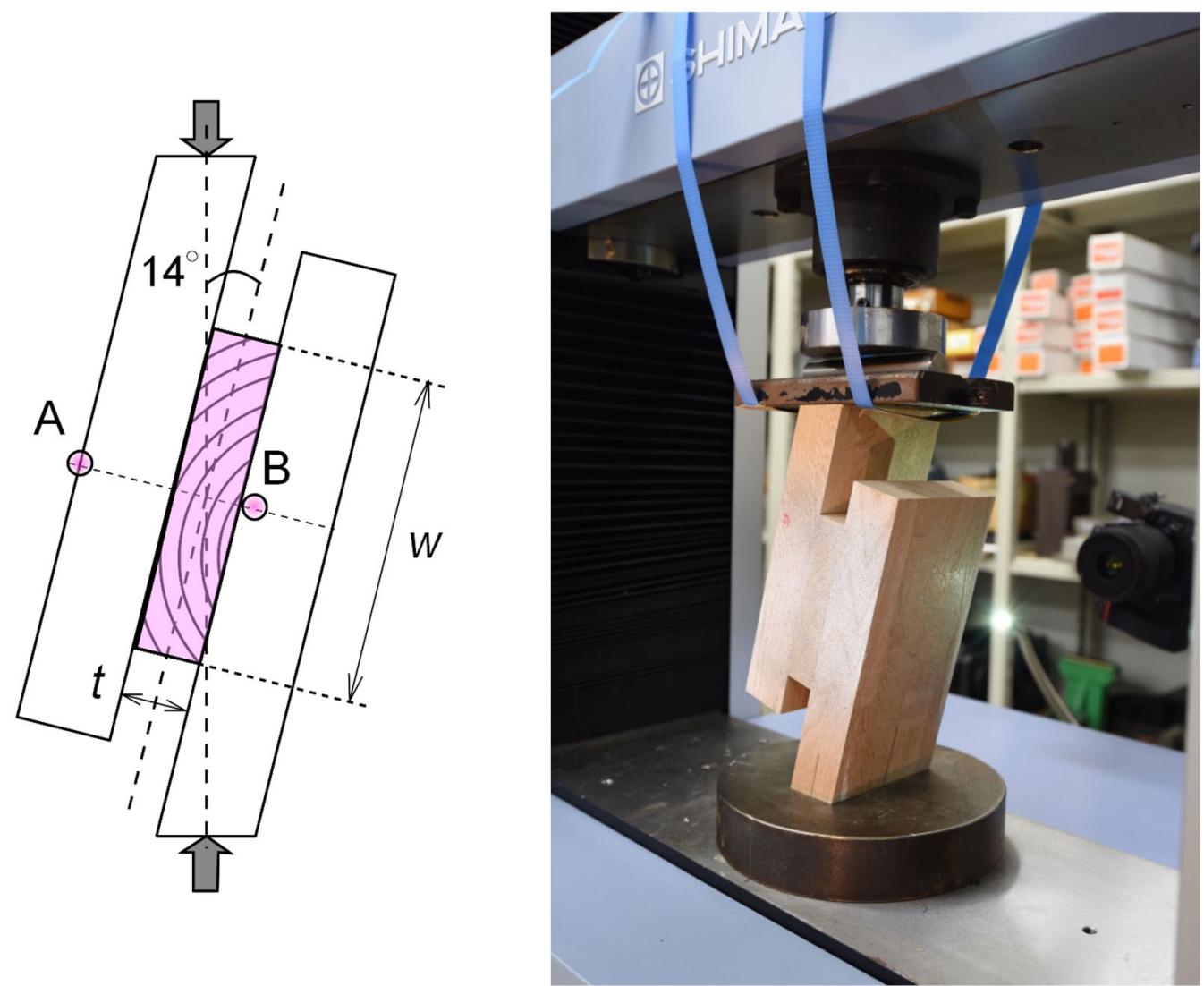

Fig. 2 Loading configuration. Points A and B indicate the locations of relative displacement measurement

first masked with white paint and airbrush-sprayed with black paint.

The samples were loaded in a universal testing machine (Shimadzu, capacity of $50 \mathrm{kN}$ ) under compression (see Fig. 2). A drift bolt was placed between the loading plate and the sample to avoid uneven loading and to allow in-plane rotation of the hardwood plate. The samples were loaded at a constant displacement of $0.3 \mathrm{~mm} / \mathrm{min}$. To allow the observation of both faces of the sample, two digital cameras (Nikon D810) were set on each side. Images were acquired continuously at 2 -s intervals. The interval was equivalent to $0.01 \mathrm{~mm}$ of crosshead displacement. Using a data acquisition module (National Instruments NI 9234), the load value at the time of image acquisition was recorded. An example of an acquired image is presented in Fig. 3. The distance between the sample and the surface of the charge-coupled device (CCD) chip in the camera was adjusted during each test to maintain a constant spatial resolution. The average spatial resolution was set at $0.025 \mathrm{~mm} /$ pixel for all sample measurements.

As illustrated in Fig. 3, measurement of the displacement region was set to cover at least a quarter of the width of the hardwood plates and the entire cross-section of the laminae. In-plane displacement fields were obtained using VIC-2D (ver.2009, Correlate Solutions), a digital image correlation software. The displacement field consisted of grid points arranged at 5 pixels $\times 5$ pixels intervals. To calculate the displacement at each point, a subset of 31 pixels $\times 31$ pixels was used (see Fig. 3 ). The displacement field data were converted into strain values.

Generally, to measure the relative displacement of side plates, a displacement transducer is set on one plate, and a target is set on the other plate. In a strict sense, the displacement measured in this manner also contains shear deformation of the glue line. To evaluate the shear strain measurement method, two different calculation methods were compared: the shear strain calculated from the relative displacements of the side plates and the average shear strain in the cross-section of laminae. Relative displacement was measured based on the distance between two points on the side plates (refer to Fig. 2). The shear modulus based on the relative displacements $\left(G_{\mathrm{dsp}}\right)$ and the shear modulus obtained from the strain field $\left(G_{\text {str }}\right)$ were calculated using the following equations: 


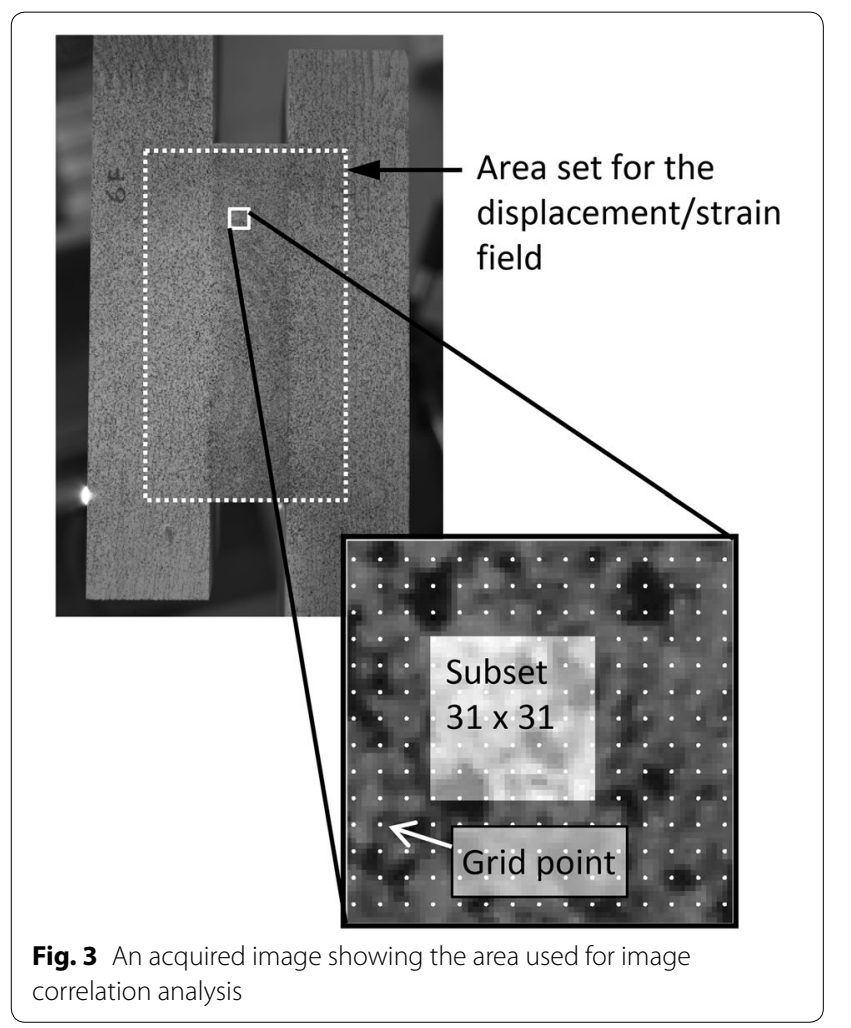

$$
\begin{aligned}
& G_{\mathrm{dsp}}=\frac{\Delta P \cos 14^{\circ}}{w L} \cdot \frac{t}{\Delta d}, \\
& G_{\mathrm{str}}=\frac{\Delta P \cos 14^{\circ}}{w L} \cdot \frac{1}{\Delta \gamma},
\end{aligned}
$$

where $\Delta P$ is the increment in load $(\mathrm{N}), t$ the thickness of the lamina $(\mathrm{mm}), w$ the width of the lamina $(\mathrm{mm}), L$ the longitudinal length of the lamina $(\mathrm{mm}), \Delta d$ the increment in the relative displacement of the side plate $(\mathrm{mm})$, and $\Delta \gamma$ the increment in the average strain of a lamina. Rolling shear strength $\left(f_{\mathrm{r}}\right)$ was calculated using the following equation:

$$
f_{\mathrm{r}}=\frac{P_{\max } \cos 14^{\circ}}{w L},
$$

where $P_{\max }$ is the maximum load $(\mathrm{N})$ and $w$ and $L$ are similar to the cases in Eqs. (1) and (2).

\section{Theoretical background and numerical calculation of the apparent rolling shear modulus of the lamina}

The shear modulus of Japanese cedar in the rolling shear direction $\left(G_{\mathrm{RT}}\right)$ has been reported as $15 \mathrm{MPa}$ in previous literature $[9,10]$. The value was obtained using the following equation for rotation of the on-axis Hooke's law to an

\begin{tabular}{|c|c|c|c|c|c|}
\hline $\begin{array}{l}\text { Density }{ }^{a}(\mathrm{~kg} / \\
\left.\mathrm{m}^{3}\right)\end{array}$ & $E_{\mathrm{T}}(\mathrm{MPa})$ & $E_{\mathrm{R}}(\mathrm{MPa})$ & $E_{45^{\circ}-\mathrm{RT}}(\mathrm{MPa})$ & $\boldsymbol{v}_{\mathrm{TR}}$ & $G_{\mathrm{RT}}(\mathrm{MPa})$ \\
\hline 330 & 290 & 590 & 60 & 0.44 & 15 \\
\hline \multicolumn{6}{|l|}{ Air-dry density } \\
\hline 1 & 4 & 1 & $2 v_{\mathrm{RT}}$ & & \\
\hline$\overline{G_{R T}}$ & $\overline{E_{45^{\circ}-\mathrm{RT}}}$ & $\overline{E_{\mathrm{R}}}$ & $\overline{E_{\mathrm{T}}}+$ & & \\
\hline
\end{tabular}
off-axis direction:
Table 1 Elastic constants of Japanese cedar [9]

where $G_{\mathrm{RT}}$ is the rolling shear modulus in the RT-plane, $E_{45^{\circ}-\mathrm{RT}}$ the modulus of elasticity in the $45^{\circ}$ rotated offaxis direction, and $E_{\mathrm{R}}$ and $E_{\mathrm{T}}$ are the moduli of elasticity in the radial and tangential directions, respectively. $\nu_{\mathrm{RT}}$ is the Poisson's ratio. The rolling shear modulus in the RTplane can be calculated by substituting the experimentally measured modulus of elasticity in the RT $45^{\circ}$ off-axis direction and Poisson's ratio. Each modulus of elasticity was obtained using a compression test of prismatic bar specimens [10]. In Table 1, the elastic moduli obtained in the compression test, air-dry density, and calculated $G_{\mathrm{RT}}$ are listed $[9,10]$.

The value of $G_{\mathrm{RT}}=15 \mathrm{MPa}$ reported in the literature [9] is low compared to the value of shear modulus of the lamina observed in the present study. However, if the polar anisotropic features of wood are taken into account, the local shear modulus in a lamina could be greater than pure $G_{\mathrm{RT}}$. By applying a similar rotational transformation operation to the rolling shear $G_{\mathrm{RT}}$, the shear modulus in an arbitrary direction on the RT-plane can be calculated using the following equation:

$$
\frac{1}{G_{\theta}-\mathrm{RT}}=4 m^{2} n^{2}\left(\frac{1}{E_{\mathrm{R}}}+\frac{1}{E_{\mathrm{T}}}+\frac{2 v_{\mathrm{RT}}}{E_{\mathrm{R}}}\right)+\frac{m^{4}+n^{4}-2 m^{2} n^{2}}{G_{\mathrm{RT}}}
$$

$$
m=\sin \theta, n=\cos \theta,
$$

where $G_{\theta-\mathrm{RT}}$ is the rolling shear modulus in an arbitrary direction on the RT-plane. The calculated relation curve of $G_{\theta-\mathrm{RT}}$ and off-axis rotation angle $\theta$ is shown in Fig. 4 . The maximum value is observed at $\theta=45^{\circ}$, and the transformed value $\left(G_{45^{\circ}-\mathrm{RT}}\right)$ was about eightfold higher than the $G_{R T}$ value. The relationship suggests that the shear modulus inside a lamina is distributed according to the local orientation of the annual ring and that the overall shear modulus of a lamina is greater than the on-axis $G_{\text {RT. }}$. To quantitatively assess the polar anisotropic effect on the shear modulus of the lamina, linear finite element analysis was conducted. Finite element analysis was performed under plane stress conditions using a triangular element (Fig. 5). Finite element models were established and analyzed using the open-source MATLAB program coded by Komatsu [11]. 

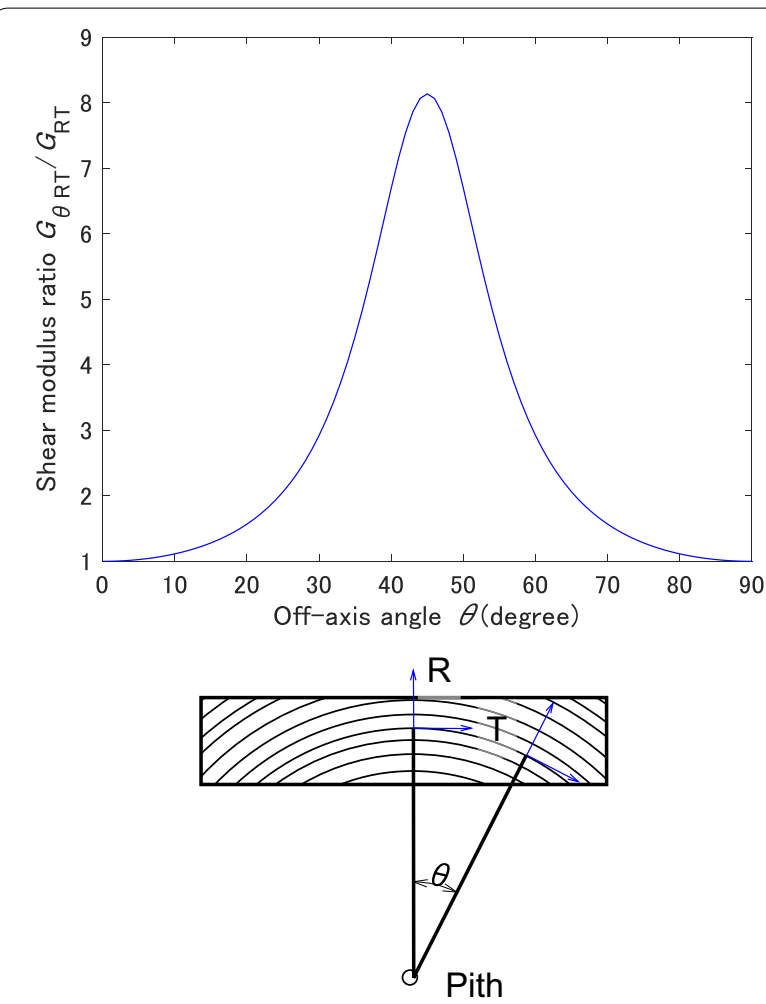

Fig. 4 Relationship between off-axis angle and shear modulus. The vertical axis is normalized with the on-axis $G_{R T}$

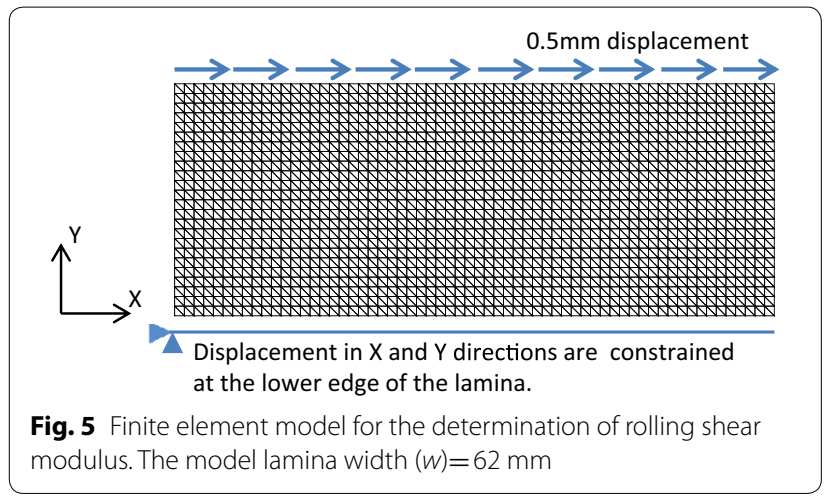

To compare the experimentally obtained shear modulus in the lamina with the previously reported on-axis shear modulus of Japanese cedar, material properties from the literature [9] were adopted (listed in Table 1). Individual triangular elements were given a stiffness matrix with the direction of the material coordinate changed according to the position from the pith. Using the coordinates of estimated pith locations, 98 (one specimen out of 99 specimens was omitted because the pith location could not be determined) specimen geometries were analyzed. In the present study, onaxis material properties were assumed to be constant regardless of the distance from the pith. Therefore, the results of the analysis are influenced solely by the geometric factor of the annual ring.

The translational movements of the nodes at the lower edge of the lamina were constrained. A forced displacement of $0.5 \mathrm{~mm}$ was applied on the nodes along the upper edge of the lamina. By substituting the obtained reaction force and forced displacement to Eq. (1), the apparent rolling shear modulus of the lamina was determined. The element division size was unified to $1 \mathrm{~mm}$. To eliminate the influence of the directionality of the triangular element, analyses were performed using two types of mesh: right-upward triangular division and left-upward triangular division. The results obtained from both analyses were averaged.

\section{Results and discussion}

\section{Comparison of measurement methods for determining rolling shear modulus}

Figure 6 presents an example of the relationships determined between shear stress and strain. Because the displacement/strain was measured using a digital camera from both faces of the sample, two stress-strain relationship curves were obtained for each method of calculation. Shear modulus was obtained from the slope with $10 \%$ to $40 \%$ of the maximum shear stress. The values obtained from both faces were averaged.

Figure 7 shows the relationships between two types of shear modulus. A highly linear relationship was found between the shear moduli based on the areal strain of laminae $\left(G_{\text {str }}\right)$ and shear moduli based on the relative displacement of the side plates $\left(G_{\mathrm{dsp}}\right)$. The slope of the regression for all data was $1.16\left(R^{2}=0.96\right) . G_{\text {str }}$ was about $16 \%$ higher than $G_{\mathrm{dsp}}$. When the difference in

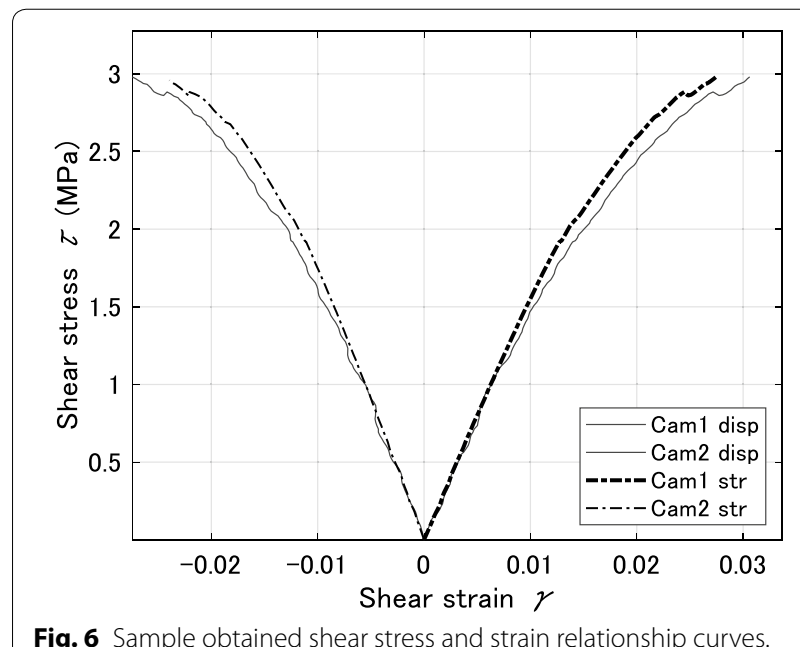

The width of the lamina was $(w)=112 \mathrm{~mm}$ 


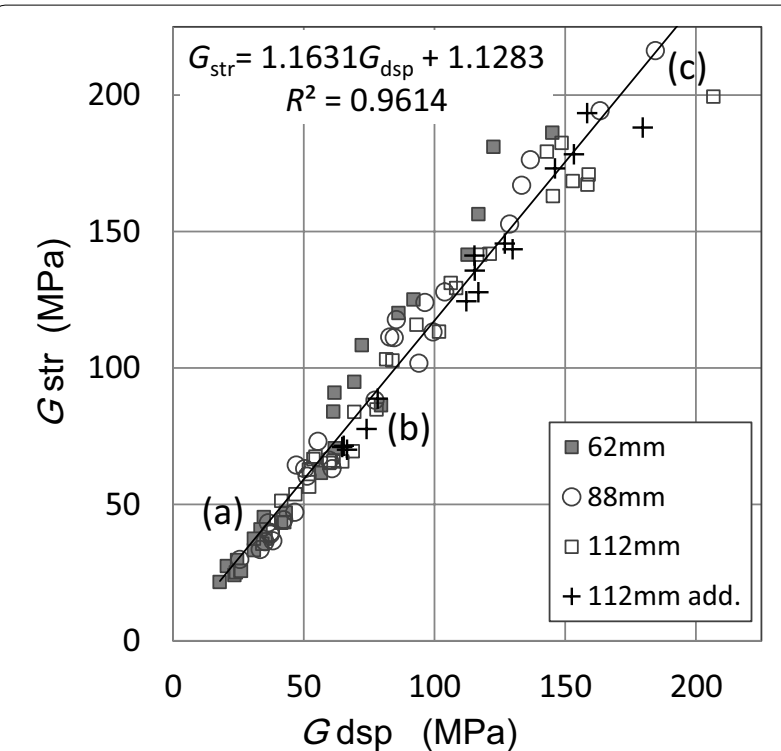

Fig. 7 Comparison of the results from two different methods for measuring rolling shear modulus. Solid squares: lamina width $w=62 \mathrm{~mm}$; circles: $w=88 \mathrm{~mm}$; open squares: $w=112 \mathrm{~mm}$, plus sings: $W=112 \mathrm{~mm}$ additional samples

shear modulus between the two methods was converted into the difference in shear strain, the shear strain in the glue lines constituted $14 \%$ of the total shear strain obtained from the relative displacement of side plates. The result indicates that, to obtain the shear modulus of the lamina alone, the areal strain inside the lamina needs to be measured. It also suggests that the application of glue in the test sample has to be realistic in order to measure the apparent rolling shear modulus, including the deformation in the interface glue layer.

The relationships between the rolling shear modulus and strength in all samples are illustrated in Fig. 8. Both types of shear moduli were highly correlated to shear strength. No relationships were observed between densities and rolling shear properties $\left(R^{2}<0.05\right)$.

\section{Influence of annual ring geometry on rolling shear properties}

For comparison, the shear strain distributions of different shear moduli $\left(G_{\text {str }}\right)$ at (a) 30 , (b) 88 , and (c) $216 \mathrm{MPa}$ were selected from the $88-\mathrm{mm}$ width data set (Fig. 9). In sample (a), a large strain was distributed throughout the lamina. In sample (b), a large strain was concentrated over half of the lamina, whereas in sample (c), the absolute strain value was low all over the lamina, and the region with the large strain was relatively small compared to those in samples (a) and (b).

The estimated locations of the pith for each of the samples from (a) - (c) are illustrated in Fig. 9. The order

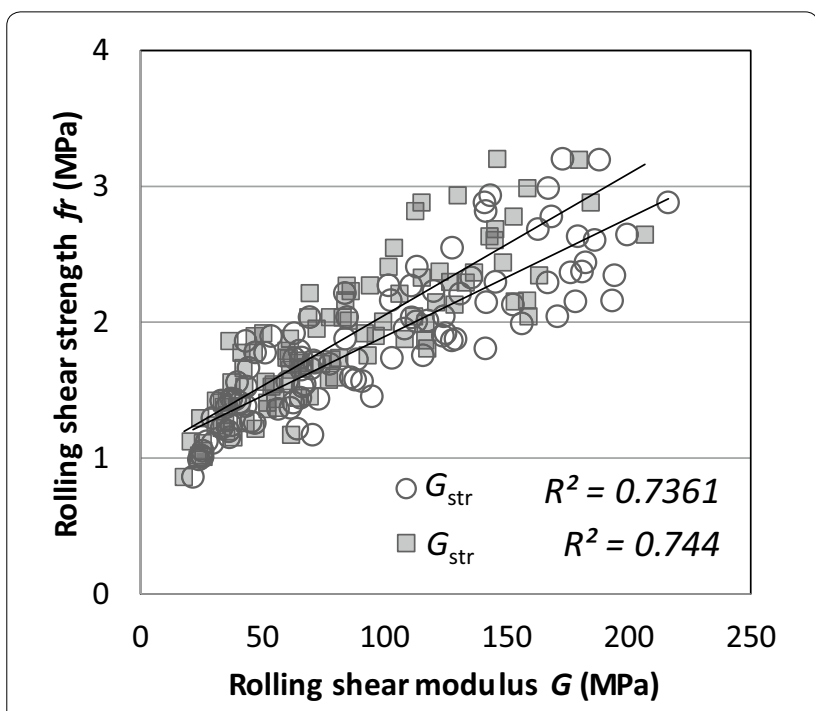

Fig. 8 Correlation between rolling shear strength and shear modulus

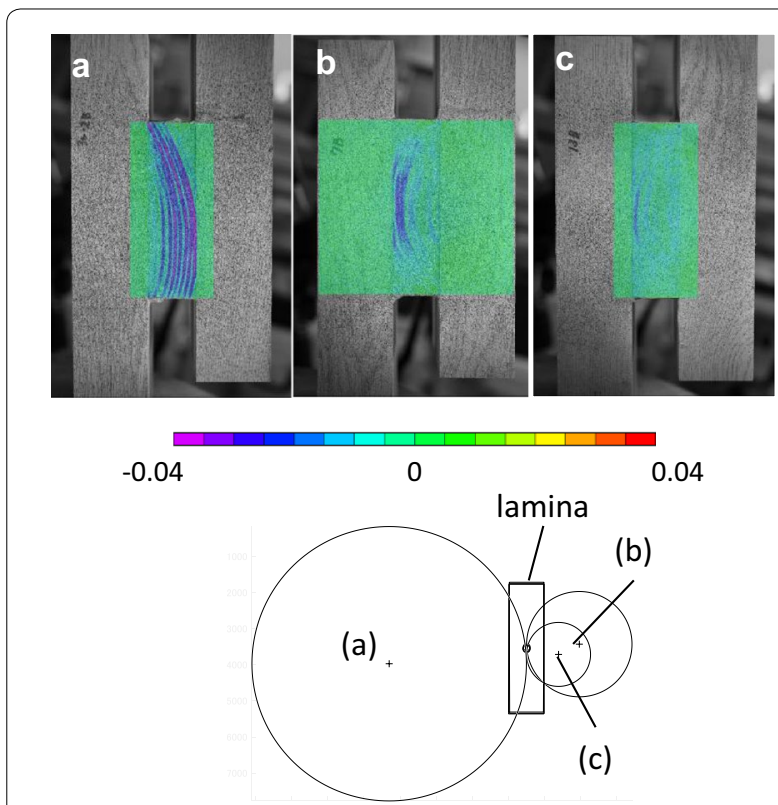

Fig. 9 Comparison of shear strain distributions with different annual ring geometries at $88-\mathrm{mm}$ width compared at $40 \%$ of maximum load. The lower diagram illustrates the estimated locations of the pith for (a), (b), and (c). Refer to Fig. 7 for the shear modulus of (a), (b), and (c)

of the distances from the pith to the center of the lamina was $(\mathrm{c})<(\mathrm{b})<(\mathrm{a})$. The large shear-strain region roughly matched with the location where the curvature of the annual ring is low and with the location where the tangential direction of the annual ring was parallel to the shear force. In addition, strain distribution was greatly influenced by the annual ring orientation in the cross-section. 

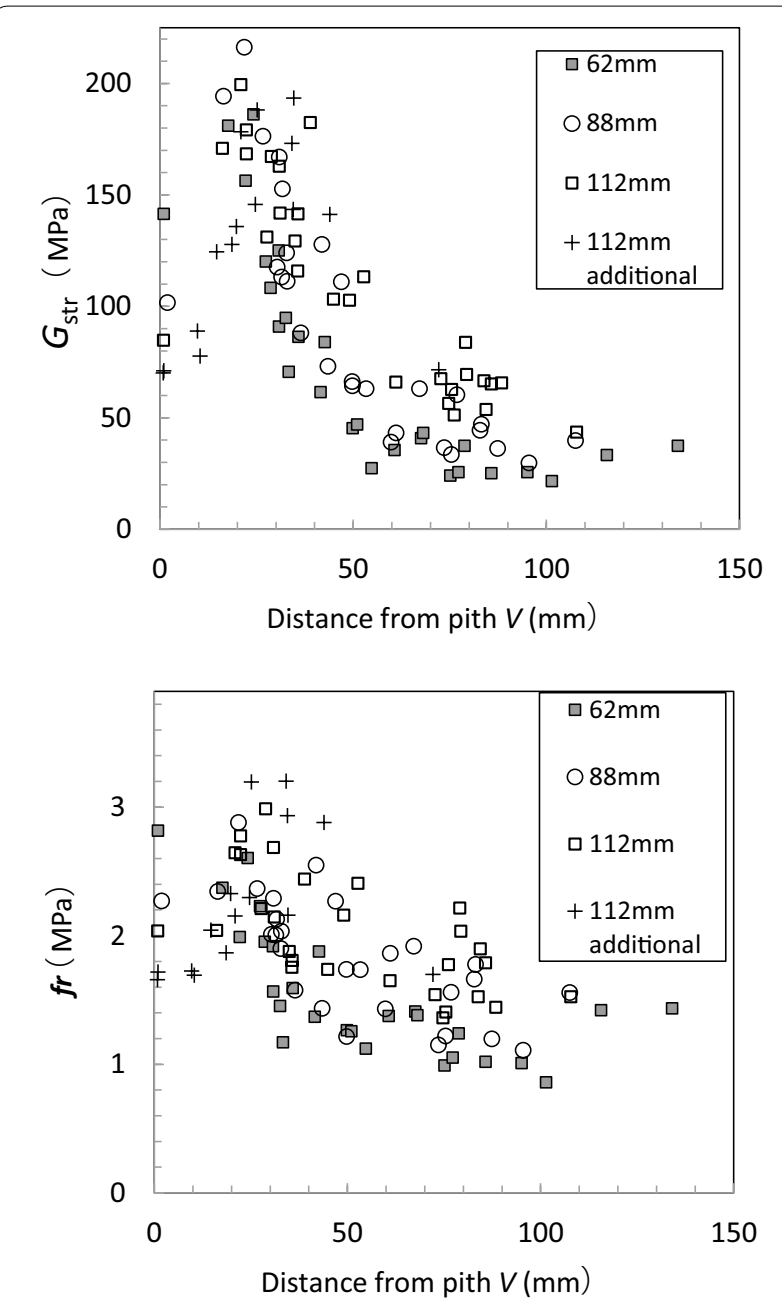

Fig. 10 Influence of distance from pith ( $V$ ) on rolling shear modulus $\left(G_{\text {str }}\right)$ and strength $\left(f_{\mathrm{r}}\right)$. Solid squares: lamina width $w=62 \mathrm{~mm}$; circles: $w=88 \mathrm{~mm}$; open squares: $w=112 \mathrm{~mm}$, plus signs: $w=112 \mathrm{~mm}$ additional samples

Figure 10 shows the effect of distance from the pith on shear modulus and shear strength. When the samples containing pith were excluded, the shear modulus decreased with an increase in distance from the pith $(V)$. The relationship between $G_{\text {str }}$ and $V$ was nonlinear. Ehrhart et al. [2] reported that radial distances of 30, 60, and $100 \mathrm{~mm}$ did not influence the rolling shear strength of Norway spruce. In the present study, shear strength also decreased with an increase in the distance from the pith, although this was not as apparent in the case of the shear modulus.

\section{Rolling shear modulus obtained in numerical analysis}

Figure 11 shows the effect of distance from the pith $(V)$ on the numerically calculated shear modulus $\left(G_{\mathrm{fem}}\right)$.
Similar trends were observed as seen in the experiment: the lamina that included a pith in the cross-section had a low shear modulus (data enclosed with a dotted line in the figure), and shear modulus decreased nonlinearly with an increase in distance from the pith $(V)$. As the distance from the pith increased, the rolling shear modulus of a lamina approached the on-axis shear modulus $G_{\mathrm{RT}}$. The results imply that the lower bound of the rolling shear modulus of a lamina is nearly equal to the on-axis shear modulus $G_{\mathrm{RT}}$.

Although it was not considerable in the results of the experiment, in the sample without the pith, $G_{\text {fem }}$ demonstrated a slight increase with an increase in lamina width $(w)$.

The relationship between the calculated shear modulus $\left(G_{\mathrm{fem}}\right)$ and the measured shear modulus $\left(G_{\mathrm{str}}\right)$ is shown in Fig. 12. Sample groups with the pith and without the pith displayed different trends. In the sample group without the pith, $G_{\text {fem }}$ was nonlinearly correlated with $G_{\text {str }}$. However, the range of the calculated shear modulus $G_{\text {fem }}(22$ to $93 \mathrm{MPa}$ ) and the range of measured $G_{\text {str }}(22$ to $216 \mathrm{MPa})$ did not match in the upper bound. In the sample group with the pith, the measured value $\left(G_{\text {str }}\right)$ was high compared to the relatively low $G_{\text {fem }}$ value.

Shear strain distributions in the numerical analysis and the experiment were compared for different distances from the pith (Fig. 13). As observed in the contour maps and the vertical and horizontal line profiles in Fig. 13, the distribution trends in the numerical analysis and the experiment were somewhat consistent, although the actual strain distribution was influenced considerably by the earlywood/

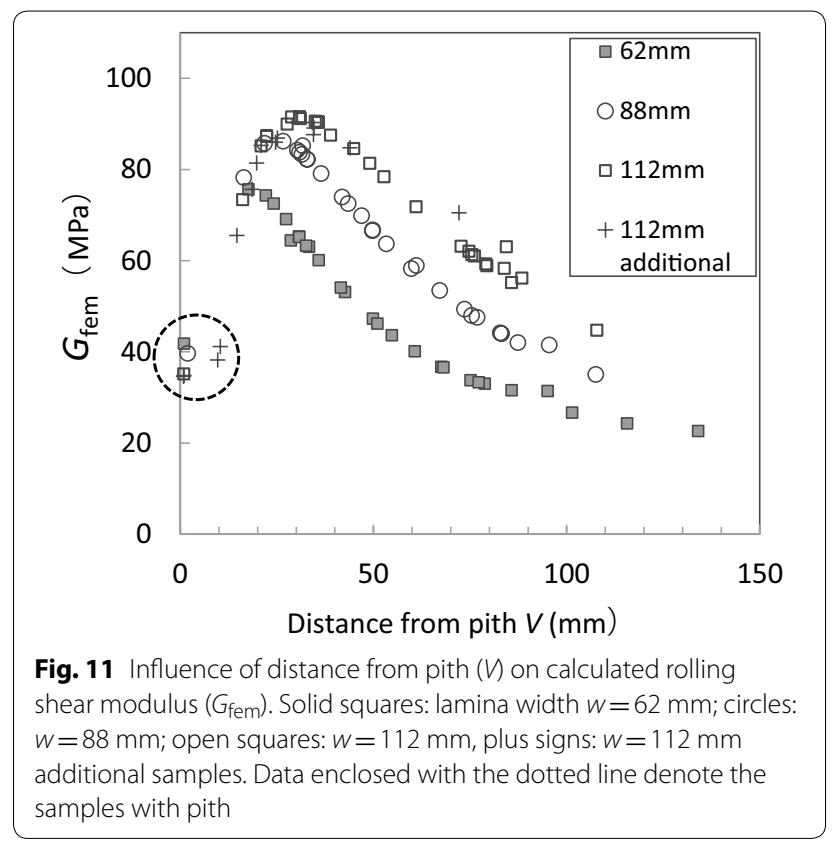




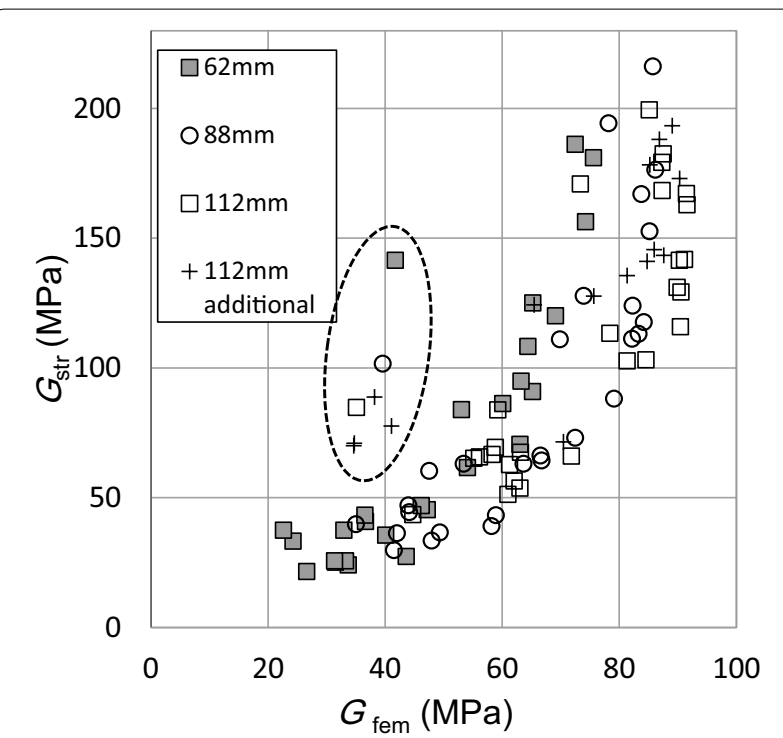

Fig. 12 The relationship between the measured and calculated shear moduli. Solid squares: lamina width $w=62 \mathrm{~mm}$; circles: $w=88 \mathrm{~mm}$; open squares: $w=112 \mathrm{~mm}$; plus signs: $w=112 \mathrm{~mm}$ additional samples. Data enclosed with the dotted line denote the samples with pith

latewood structures. The cause of the difference in the upper bound of $G_{\text {str }}$ and $G_{\text {fem }}$ can be observed in the strain distributions near the pith (examples 1 and 2 in Fig. 13), where the strain value measured in the experiment was significantly low compared to that in the analysis, which suggested that the local shear modulus near the pith was higher than the uniformly set shear modulus in the numerical analysis. To bring the analysis results closer to the experimental results, the influence of the variation in the properties of the material in the radial direction requires further investigation, which, however, is outside the scope of this paper.

\section{Influence of lamina width on rolling shear properties}

The statistical values obtained in the present study are summarized in Table 2 for the respective lamina widths $(w): 62,88$, and $112 \mathrm{~mm}$. The mean shear modulus and strength demonstrated a slight increase with an increase in lamina width $(w)$. The coefficients of variation $(\mathrm{CV})$ for shear modulus and strength decreased with an increase in lamina width. Notably, the CV of the calculated value of shear modulus $\left(G_{\text {fem }}\right)$ also showed a trend similar to the measured shear modulus. The observed CV trend here highly suggests that the variance in shear modulus was caused by the geometric factor in the annual ring structure. Okabe et al. [5] reported a rolling shear strength of 1.64 MPa for Japanese cedar laminae for single lamina rolling shear strength, where the cross-section was $74 \mathrm{~mm}$ wide and $30 \mathrm{~mm}$ thick (the aspect ratio $w / t=2.47$ ). In the present study, the mean value was $1.54 \mathrm{MPa}$ for a $62-\mathrm{mm}$ wide (the aspect ratio $w / t=2.58$ ) cross-section, which is comparable with the result of Okabe et al. [5]. Figure 14 shows the cumulative distribution of shear modulus and strength. Lognormal cumulative distribution curves were fitted to calculate the $5 \%$ quantiles. The $5 \%$ quantile of the rolling shear properties also demonstrated an increase with an increase in lamina width.

\section{Conclusion}

A shear test configuration similar to the shear test prescribed in the EN 408 standard was conducted to obtain the rolling shear properties of Japanese cedar with different lamina sizes.

The rolling shear modulus based on the areal strain of laminae $\left(G_{\text {str }}\right)$ was $16 \%$ higher than the shear modulus based on the relative displacement of the side plates $\left(G_{\mathrm{dsp}}\right)$.

The value of the rolling shear modulus and strength decreased with an increase in radial distance from the pith. Regarding the shear modulus, the estimation obtained using the continuum mechanics showed that the value and its variance were influenced substantially by the annual ring structure. Based on the analysis, it was deduced that the shear modulus in lamina size asymptotically approaches the on-axis shear modulus $G_{\text {RT }}$ with an increase in radial distance from the pith.

The mean rolling shear modulus was $72 \mathrm{MPa}$ for the $62-\mathrm{mm}$ wide and $24-\mathrm{mm}$ thick value. The value was greater than the on-axis rolling shear modulus of $G_{\mathrm{RT}}=15 \mathrm{MPa}$, which has previously been reported for 
a Original scanned image

\section{Example 1}

Distance from pith $V=1 \mathrm{~mm}$

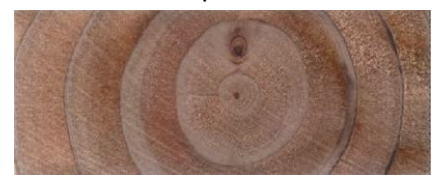

Example 2

Distance from pith $V=24 \mathrm{~mm}$

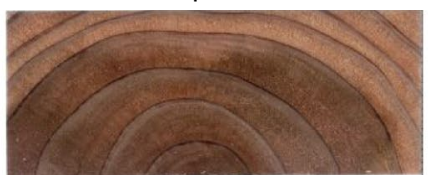

Example 3

Distance from pith $V=134 \mathrm{~mm}$

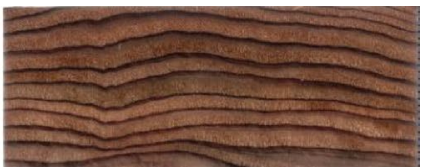

b Measured strain distribution (DIC).
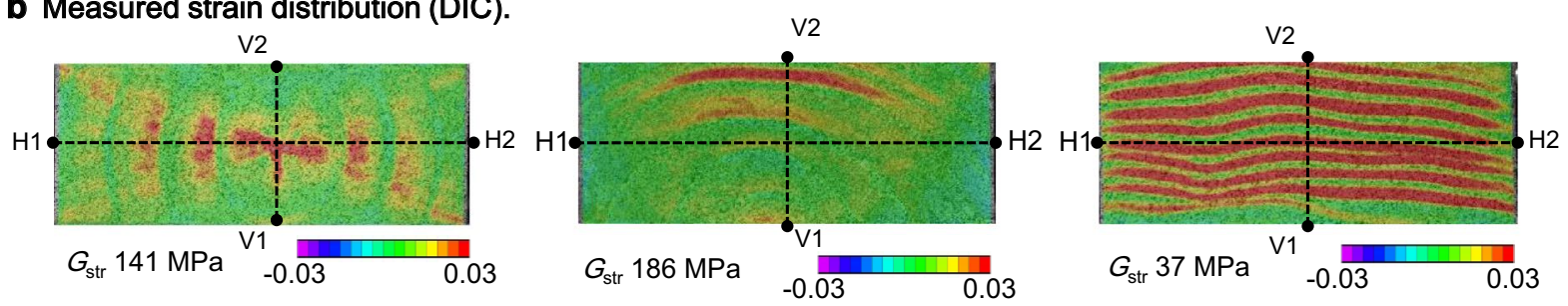

c Numerically obtained strain distribution (FEM)
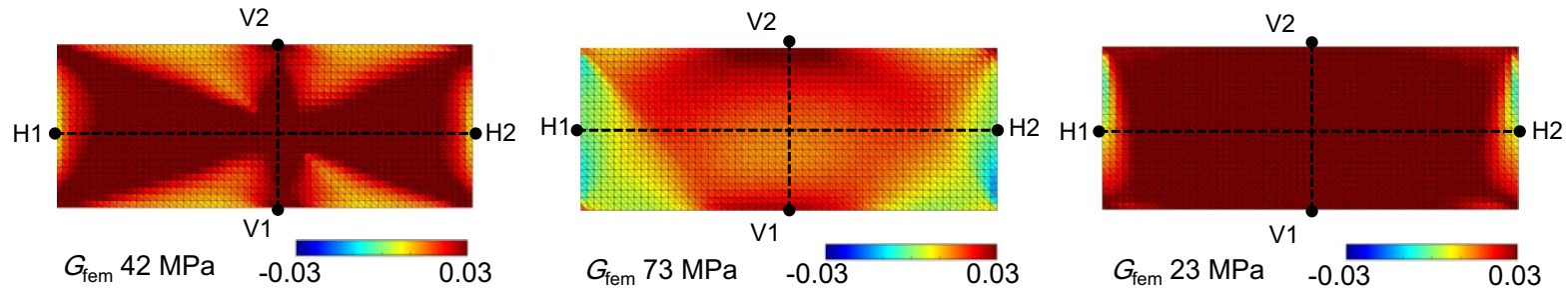

d Comparison of strain distributions along vertical (V1-V2) and horizontal (H1-H2) lines
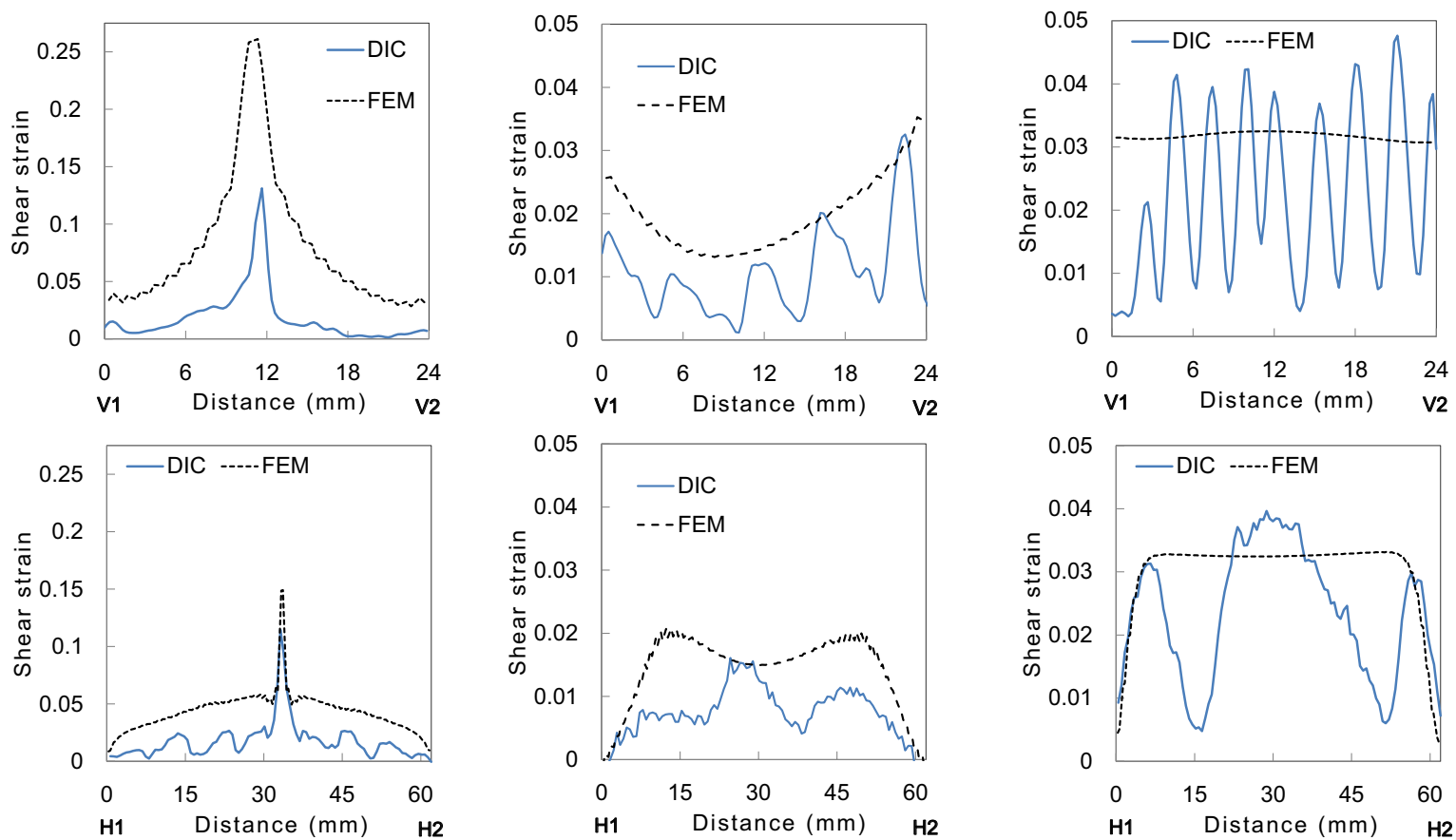

Fig. 13 Comparison of shear strain distributions in the numerical analysis and in the experiment. Lamina width $(w)=62 \mathrm{~mm}$. Strain distributions were compared at 50\% the maximum load. Contour plots of finite element analyses were adjusted to the same load levels as the experiment 
Table 2 List of sample characteristics and the observed shear properties

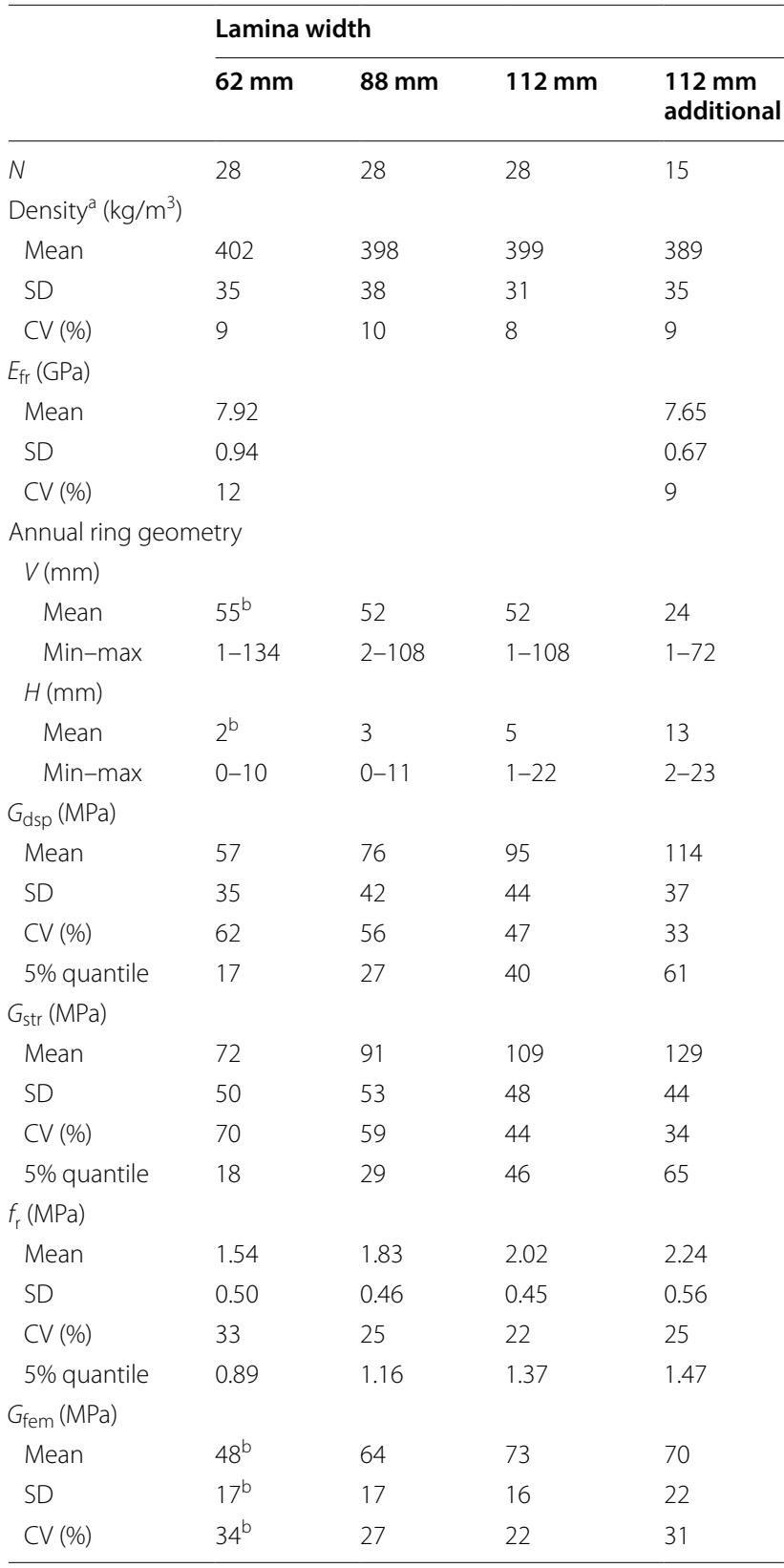

${ }^{a}$ Air-dry density

${ }^{\text {b }} N=27$. One sample was unable to determine the pith position

Japanese cedar [9]. The mean shear strength for 62-mm wide samples was $1.54 \mathrm{MPa}$, which was comparable to the results reported in a previous study conducted on Japanese cedar [5]. The mean values of shear modulus and strength, in addition to the $5 \%$ quantile, increased with an increase in lamina width.
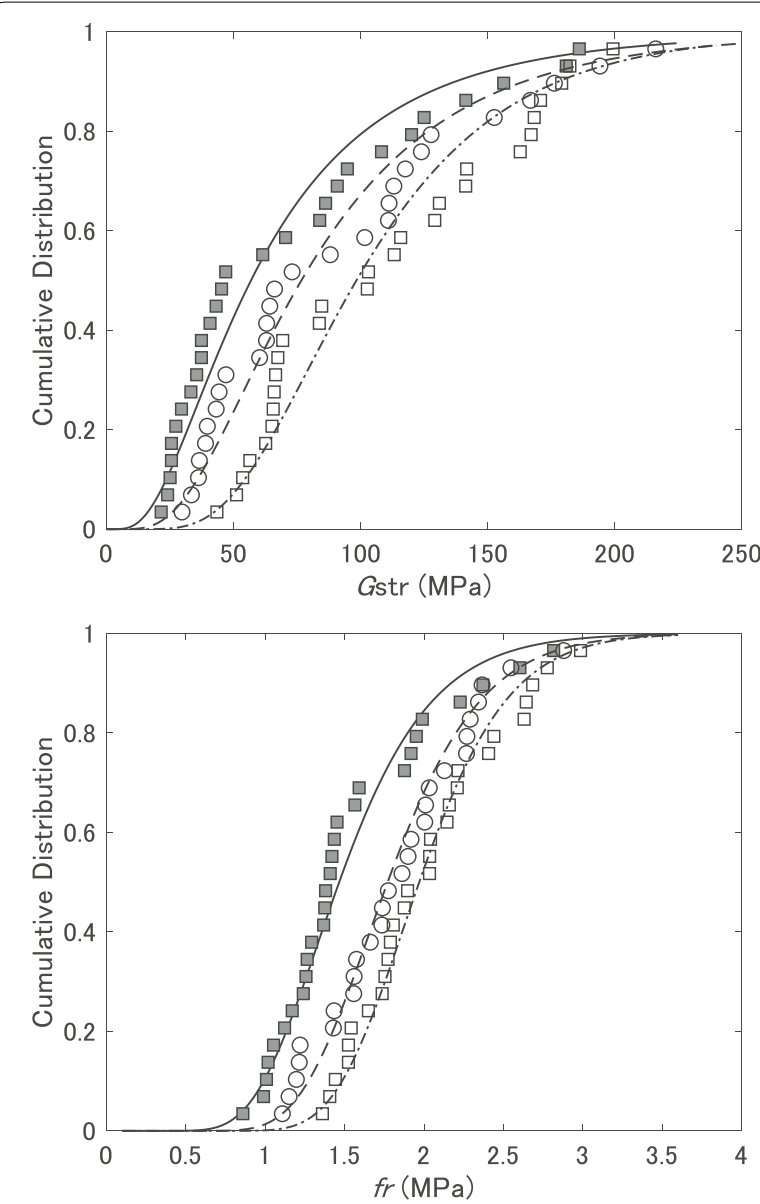

Fig. 14 Cumulative distribution of rolling shear modulus $\left(G_{\text {str }}\right)$ and shear strength $\left(f_{\mathrm{r}}\right)$. Solid squares: lamina width $w=62 \mathrm{~mm}$; circles: $w=88 \mathrm{~mm}$; open squares: $w=112 \mathrm{~mm}$

\begin{abstract}
Abbreviations
CLT: cross-laminated timber; $E_{\mathrm{fr}}$ elastic modulus in the direction parallel to the grain, i.e., longitudinal, direction; $V$ : radial distance to the pith; $H$ : tangential distance to the pith; MC: moisture content; CCD: charge-coupled device; $w$ : width of a lamina; $L$ : longitudinal length of a lamina; $t$ : thickness of a lamina; $\triangle P$ : increment in load; $P_{\max }$ : maximum load; $G_{\mathrm{dsp}}$ : shear modulus based on the relative displacements of side plates; $G_{\text {str: }}$ shear modulus obtained from strain field; $f_{r}$ : rolling shear strength; $\Delta d$ : increment in the relative displacement; $\Delta y$ : increment in the average strain of a lamina; $G_{R T}$ : shear modulus in the radial-tangential plane (rolling shear); $E_{\mathrm{R}}$ : modulus of elasticity in the radial direction; $E_{\mathrm{T}}$ : modulus of elasticity in the tangential direction; $E_{45^{\circ}-\mathrm{RT}}$ : modulus of elasticity in the $45^{\circ}$ rotated off-axis direction; $V_{R T}$ : Poisson's ratio in the radial-tangential plane; $G_{\theta-R T}$ : rolling shear modulus in an arbitrary direction on the RT-plane; $\theta$ : off-axis rotation angle; $\mathrm{CV}$ : coefficients of variation; SD: standard deviation.
\end{abstract}

\section{Acknowledgements}

We thank Watanabe K. of the Forestry and Forest Products Research Institute for his advice on the statistical analysis.

\section{Authors' contributions}

SU performed digital image correlation and numerical analysis, and was a major contributor in writing the manuscript. SK designed the shear test configurations and contributed to the improvement of the test set-up. MA 
contributed to the analysis and interpretation of the data. All authors read and approved the final manuscript.

\section{Funding}

This work was supported by JSPS KAKENHI Grant Number 15 K07521.

\section{Availability of data and materials}

The datasets used and analyzed in the current study are available from the corresponding author on reasonable request.

\section{Competing interests}

The authors declare that they have no competing interests.

Received: 6 January 2019 Accepted: 26 June 2019

Published online: 08 July 2019

\section{References}

1. Aicher S, Dill-Langer G (2000) Basic considerations to rolling shear modulus in wooden boards. Otto-Graf-J 11:157-165

2. Ehrhart T, Brandner R, Shickhofer G, Frangi A (2015) Rolling shear properties of some European timber species with focus on cross laminated timber (CLT): test configuration and parameter study. In: Conference paper of international network on timber engineering research (INTER), Volume: 2nd Meeting, Sibenik, Croatia, 24-27 August 2015

3. EN Standard 408 (2012) Timber structure-structural timber and glued laminated timber-determination of some physical and mechanical properties. European Committee for Standardization, Brussels
4. Aicher S, Chirstian Z, Hirsch M (2016) Rolling shear modulus and strength of beech wood laminations. Holzforschung 70(8):773-781

5. Okabe M, Yasumura M, Kobayashi K (2014) Prediction of internal shear capacity of Sugi CLT panels. Mokuzai Gakkaishi 60(3):169-176

6. EN Standard 314-1 (2004) Plywood_bonding quality_Part 1: test methods. European Committee for Standardization, Brussels

7. Richard B (2007) https://jp.mathworks.com/matlabcentral/fileexchan ge/15060-fitcircle-m. Accessed 7 Nov 2017

8. Gander W, Golub Strebel R (1994) Least-squares fitting of circles and ellipses. BIT Num Math 34:558-578

9. Forestry and Forest Products Research Institute (2004) Mokuzai Kogyou Handbook, Maruzen, p 135 (in Japanese)

10. Yamai R (1959) Studies on the orthotropic properties of wood in compression. Bulletin of the Forestry and Forest Products Research Institute No. 113, pp 58-102

11. Komatsu K (2013) https://www.morikita.co.jp/books/book/2676. Accessed 10 May 2019

\section{Publisher's Note}

Springer Nature remains neutral with regard to jurisdictional claims in published maps and institutional affiliations. 